\title{
Magistrats et occupants, quelques jalons d'une comparaison diachronique (1914-1918 / 1940-1945)
}

\author{
Mélanie Bost \& Kirsten Peters
}

Mélanie Bost : Mélanie Bost a étudié I'histoire moderne et les arts non-européens à l'Université libre de Bruxelles. Depuis mai 2013, elle est détentrice d'un doctorat en histoire (UCL) pour une thèse portant sur l'attitude la magistrature belge pendant la Première Guerre mondiale. Dans le cadre du PAI « Justice et Populations », elle poursuit ses recherches sur l'impact de la Première Guerre mondiale sur le système judiciaire belge. Ses autres centres intérêts sont l'histoire du renseignement, I'histoire sociale des sociétés occupées en 1914-1918 et les commémorations de la Première Guerre mondiale. Elle est aujourd'hui attachée au Service général du Pilotage du Système éducatif de la Fédération Wallonie-Bruxelles.

Kirsten Peters : Kirsten Peters est licenciée en Histoire. Elle est aussi candidate en ethnologie et guide touristique graduée. Actuellement, elle est doctorante aux Facultés universitaires Saint-Louis. Dans le cadre du projet du Pôle d'attraction interuniversitaire P6/01 “Justice and Society :

Sociopolitical History of Justice Administration in Belgium (1795-2005)", elle prépare une thèse de doctorat à I'Université Saint-Louis - Bruxelles, dont le sujet porte sur la magistrature belge sous l'occupation (1940-1944).

DOI: $10.25518 / 1370-2262.397$

\section{Résumé :}

De la période française à celle de l'après-1945, la composition sociologique du corps s'avère l'une des caractéristiques les plus stables de la magistrature belge.

Les pratiques de reproduction sociale et d'endogamie, associées à un mode de recrutement fermé de la profession, ont permis l'installation de dynasties de magistrats. La stabilité politique du pays a également contribué à renforcer celle du corps judiciaire.

À deux reprises, cependant, cette stabilité a été brutalement menacée. Durant les deux guerres mondiales, l'installation d'un pouvoir étranger bouleversa l'équilibre entre les pouvoirs et malmena l'indépendance de la magistrature.

À partir de sources belges et allemandes, Mélanie Bost et Kirsten Peters comparent l'impact des deux occupations allemandes sur le corps judiciaire à partir de deux thématiques : la politique de nomination de l'occupant dans l'ordre judiciaire et les mesures de rigueur exercées contre certains de ses membres. Les moyens de défense des magistrats sont également examinés. La comparaison, jamais encore tentée pour l'histoire judiciaire, permet de pointer les spécificités et de dégager quelques invariants, tant dans la politique d'occupation que dans l'attitude des magistrats.

Mots-clés : Guerres mondiales, Histoire du droit et de la justice, Occupations militaires, Sociologie des acteurs judiciaires

\section{Abstract :}

From the French period to the post-1945 period, the sociological composition was one of the 
Magistrats et occupants, quelques jalons d'une comparaison diachronique (1914...

most stable characteristics of Belgian magistracy as a body. The practices of social reproduction and endogamy, associated with closed recruitment methods, enabled the establishment of dynasties of magistrates. The political stability of the country also contributed to strengthening that of the judicial body.

Yet, this stability was brutally threatened on two occasions. During both world wars, the establishment of a foreign power disrupted the balance of powers and jeopardised the independence of magistrates.

Based on Belgian and German sources, this paper compares the impact of German occupations on the judicial body from two perspectives : the appointment policy in the judiciary and the measures of control taken against some of its members. It also examines the means used by magistrates in their own defence.

The comparison, which has never been made before for the judicial history of Belgium, provides the possibility to identify specificities and to outline a few invariants in the occupation policy and in the behaviour of magistrates.

Keywords : History of law and justice, Military occupations, Sociology of the judicial actors, World wars

\section{Introduction 1}

De la période française étudiée par Jacques Logie $\underline{2}$ à celle de l'après-1945 analysée par Eva Schandevyla , la composition sociologique du corps s'avère l'une des caractéristiques les plus stables de la magistrature belge.

Les pratiques de reproduction sociale et d'endogamie, associées à un mode de recrutement fermé de la profession, ont permis l'installation de dynasties de magistrats. Si la politisation des nominations a pu, à certaines périodes, « colorer » le corps judiciaire, celui-ci présente cependant une forte homogénéité sociale. La plupart des magistrats qui accèdent à la haute magistrature pendant la première moitié du $20^{\mathrm{e}}$ siècle proviennent de grandes familles de la haute bourgeoisie ou de la noblesse d'expression francophone. Pour les outsiders, l'accès aux plus hautes fonctions est largement barré.

À la différence de la France, où les changements de régime brutaux et les épurations consécutives ont pu avoir pour effet un renouvellement sociologique du corps, en Belgique, après la période révolutionnaire qui a donné lieu à une vague de révocations anti-orangistes $\underline{4}$, la stabilité politique du pays a également contribué à renforcer celle du corps judiciaires 5 .

À deux reprises, cependant, cette stabilité a été brutalement menacée. Durant les deux guerres mondiales, l'installation d'un pouvoir étranger bouleversa l'équilibre entre les pouvoirs et malmena l'indépendance de la magistrature.

La présente contribution, première mise en commun des résultats de recherche de deux chercheuses actives au sein du programme PAI « Justice \& Society », propose de comparer l'impact des occupations allemandes sur le corps judiciaire à partir de deux thématiques : la politique de nomination de l'occupant dans l'ordre judiciaire et les mesures de rigueur exercées contre certains de ses membres. Les moyens de défense des magistrats sont également examinés. La comparaison, jamais encore tentée pour l'histoire judiciaire $\underline{6}$, permet de pointer les spécificités et de dégager quelques invariants, tant dans la politique d'occupation que dans l'attitude des magistrats. 
Létude repose sur des sources belges et allemandes : côté belge, elle s'appuie principalement sur les dossiers de nomination et les dossiers disciplinaires de magistrats produits par le ministère de la Justice et conservés aux Archives générales du Royaume (cité après AGR) $\underline{7}$; côté allemand, sur les rapports de l'administration civile active en 1914-1918 8 et sur ceux du Gruppe Justiz de l'administration militaire qui lui a succédé en 1940-1945ㅁ․

\section{Préambule : occupations et paysage judiciaire}

Durant les deux occupations du pays, la magistrature reste en place. Considérée comme le premier des services publics, la justice doit poursuivre son exercice. Ce maintien fait l'objet d'un large consensus parmi les auteurs de doctrine spécialistes du droit des gens (occupatio bellica) et, conformément à celle-ci, les autorités belges ont, préalablement aux invasions du territoire, rédigé des instructions préconisant ce maintien voire, pour la seconde occupation, prévu des dispositions pénales sanctionnant l'abandon de poste (loi 'Bovesse' du 5 mars 1935)ㅁ․ Globalement, la poursuite de la justice nationale convient aux occupants, soucieux du maintien de l'ordre et de la pacification du territoire : elle leur permet d'économiser leurs propres ressources.

Principal cadre juridique régissant les relations occupés-occupants, la Convention de La Haye de 1907 confie à ces derniers une mission d'administration provisoire du territoire occupé. En vertu de la convention ratifiée par les deux pays, l'occupant est responsable du maintien de l'ordre et de la vie publique et, dans l'accomplissement de sa mission, est tenu de respecter, sauf empêchement absolu, la législation du pays.

Cependant, le régime d'occupation diffère pendant les deux guerres. En 1914-1918, les autorités allemandes optent pour la continuité des services administratifs belges et la mise en place d'une administration civile chargée de chapeauter ceux-ci. Les services publics belges poursuivent la gestion des affaires courantes sous la surveillance directe des Allemands. Au sein de l'administration civile, une section Justice - la Justiz Abteilung -, composée de quelques juristes, est responsable de la surveillance du ministère de la Justice et des relations avec l'ordre judiciaire.

Avec ce dernier, pouvoir indépendant, les relations sont plus complexes. L'autorité allemande intervient de plusieurs façons dans la vie judiciaire. Substitut du pouvoir exécutif, l'administration civile adresse ponctuellement des circulaires aux procureurs généraux mais celles-ci sont peu nombreuses et le parquet belge dispose en fin de compte d'une grande autonomie pour définir sa politique pénale. Quant au gouverneur général, qui s’attribue les prérogatives royales, il exerce le pouvoir de grâce, qui lui permet d'intervenir au niveau de l'exécution des peines.

Par ailleurs, l'élément militaire allemand connait de fréquentes interactions avec la justice belge par le biais de sestribunaux, chargésde l'application de la loi martiale et de la sanction des arrêtés d'occupation11. Le champ d'action de la justice militaire allemande s'étend sans discontinuer au cours de l'Occupation, au gré de milliers d'arrêtés qui organisent le contrôle de la population et l'exploitation des ressources du territoire occupé.

Les magistrats, pour continuer d'être payés et poursuivre leurs fonctions, sont contraints de signer une déclaration de loyauté12. L'essentiel du compromis qui permet la cohabitation judiciaire tient dans cette déclaration. Les magistrats sont laissés en fonctions et sont libres de juger selon leur conscience tant qu'ils reconnaissent l'autorité allemande et les prérogatives que lui confère le droit international, et tant qu'ils s'abstiennent vis-à-vis d'elle de toute expression d'hostilité. 
Magistrats et occupants, quelques jalons d'une comparaison diachronique (1914...

Cependant, la magistrature perd une part de ses compétences au profit de la justice militaire allemande. Elle perd tout d'abord sa juridiction sur l'ensemble des individus - Allemands ou Belges - liés aux autorités d'occupation et sa compétence d'attribution est progressivement diminuée au profit des tribunaux militaires allemands.

Pendant la seconde occupation du pays, les autorités allemandes font le choix d'une administration militaire13. Celle-ci ne chapeaute plus directement les ministères belges et peut donc s'appuyer sur un personnel réduit. Pour ce qui concerne le domaine judiciaire, les missions et le profil des responsables sont cependant fort semblables à ceux de leurs prédécesseurs de la Justiz Abteilung. Les membres du Gruppe Justiz exercent la surveillance de la magistrature. Officiers de réserve, les deux responsables du service - Wilhelm van Randenborgh et Walter Hartz - sont, au civil, des magistrats professionnels.

Dès le 10 mai 1940, les intentions allemandes de maintenir les institutions belges en place et de ne s'attribuer qu'un rôle de gouvernement de surveillance sont rendues publiques en Belgique par une proclamation du commandant en chef de l'armée allemande von Brauchitsch, dans laquelle il est précisé que «les autorités locales pourront poursuivre leur activité à condition qu'elles observent une attitude loyale envers l'Armée Allemande "14. Dans sa Notice destinée à la presse dans les territoires occupés de l'Ouest, von Brauchitsch stipule que les ordonnances des chefs militaires allemands ont force de loi durant l'occupation et qu'elles « priment celles du pays » 15 .

La différence essentielle avec la première occupation allemande réside dans l'organisation des pouvoirs belges. En 1914-1918, le pouvoir judiciaire est le seul des trois pouvoirs nationaux à poursuivre son activité en territoire occupé. Le pouvoir législatif national y est suspendu. Seul l'occupant peut prendre des lois nouvelles et il est, tranche la Cour de cassation à l'issue de débats juridiques retentissants $\underline{16}$, seul juge de l'empêchement absolu de ne pas respecter les lois belges. Le contexte législatif est donc relativement clair. Il y a, d'une part, les lois nationales qui s'appliquent aussi longtemps qu'elles n'ont pas été modifiées ou abrogées par l'occupant et qui sont sanctionnées par les tribunaux belges et, d'autre part, les ordonnances d'occupation, sanctionnées par les tribunaux militaires de l'occupant. L'occupant tente parfois de faire appliquer ses ordonnances par les tribunaux belges mais, au pénal du moins, ses tentatives se soldent la plupart du temps par un échec. Les magistrats montrent beaucoup de réticences à accepter une coopération qui risquerait de les compromettre.

En 1940-1945, le paysage institutionnel est, au contraire, brouillé. Les secrétaires généraux des ministères belges, en vertu d'un système de délégations dont les limites seront sujettes à interprétation jusqu'à la fin de la guerre, exercent de façon intérimaire le pouvoir exécutif et législatif. Tout arrêté émis par ces hauts fonctionnaires belges doit cependant être préalablement soumis à l'approbation de l'occupant.

Ce système offre une excellente couverture au pouvoir allemand1․ Le Militärbefehlshaber a recours à son pouvoir exécutif dès qu'il juge les lois belges insuffisantes et émet des ordonnances qui ont force de loi. Ces ordonnances ont priorité sur le droit belge. Néanmoins, l'occupant préfère que les mesures impopulaires soient prises par les secrétaires généraux, dans l'espoir que la législation indigène soit mieux acceptée par la population18.

Le système des secrétaires généraux a cette autre conséquence essentielle qu'il crée un intermédiaire entre pouvoirs occupant et judiciaire qui n'existait pas en 1914-1918 - le chef du 
parquet général près la Cour de cassation, Georges Terlinden, était alors l'interlocuteur direct du chef de l'administration civile Maximilian von Sandt, avec qui il discutait les questions de principe. En 1940-1945, les deux pouvoirs n'interagissent que très rarement de façon directe. Ils communiquent à travers le secrétaire général du ministère de la Justice.

Une autre différence essentielle par rapport à la première occupation est la présence, en territoire belge, d'institutions du Reich autres que militaires, ce qui accentue encore la confusion. L'exemple le plus important en est la Sicherheitspolizei-Sicherheitsdienst (Sipo-SD), qui « devient pendant la guerre un synonyme de la terreur nationale-socialiste dans les territoires occupés » 19 et qui constitue un élément de menace supplémentaire pour les magistrats.

\section{Une première catégorie d'ingérence : les nominations dans l'ordre judiciaire}

\section{4-1918}

« Nous sommes au 14 septembre 1917 et nous avons toujours l'âge que nous avions le 4 août $1914 » \underline{20}$

En 1914-1918, les magistrats parviennent longtemps à repousser toute ingérence allemande dans les nominations. Bien sûr, la longueur de l'occupation confronte la magistrature à la délicate question du remplacement des personnes décédées ou atteintes par la limite d'âge. Mais, en l'absence de l'autorité légitime et pour contrer toute tentative d'intrusion du pouvoir occupant, elle élabore une stratégie discrète de remplacement, exploitant toutes les possibilités de suppléances offertes par la loi sur l'organisation judiciaire de $1869 \underline{21}$.

Bon nombre de magistrats atteignent, durant les années d'occupation, l'âge légal de la retraiteet notamment des chefs de corps2․ Sur cette question, la magistrature adopte une ligne de conduite homogène. Les magistrats repoussent spontanément leur démission à la fin de la guerre, quand le pouvoir légitime sera en mesure d'approuver leur départ et de procéder à de nouvelles nominations23. En poursuivant leurs fonctions au-delà du terme légal, ils entendent participer à l'effort patriotique.

Durant les premières années de la guerre, les autorités allemandes tolèrent cette option. Elles ne semblent pas particulièrement intéressées par les nominations ou les désignations de magistrats. La stabilité des services publics belges, en effet, est plutôt bénéfique aux Allemands. Les mises à la pension et les remplacements ont un coût qu'ils n'ont vraisemblablement pas envie de porter au budget d'une occupation non prévue dans sa durée.

La situation change dans le sillage de la séparation administrative du pays décrétée en mars 191724 . Durant l'été 1917, le pouvoir nouvellement acquis par les activistes $\underline{25}$ au ministère flamand de la Justice pousse certains de leurs partisans à proposer leur candidature aux places vacantes. C'est l'occasion pour ceux qui se savent honnis par la hiérarchie judiciaire de se créer un environnement plus favorable en implantant des magistrats flamingants $\underline{26}$.

Ainsi, dans le but de provoquer un mouvement en cascade qui libérerait des places aux échelons inférieurs, le nouveau secrétaire général Flor Heuvelmans27 demande-t-il aux procureurs généraux, dès son arrivée au ministère en juillet 1917, de lui soumettre la liste des places vacantes et de 
Magistrats et occupants, quelques jalons d'une comparaison diachronique (1914...

convoquer les cours aux fins de procéder aux présentations.

Du Havre, le gouvernement belge réagit au moyen d'un arrêté-loi qui dispose que toutes les mesures prises par l'occupant (donc aussi les nominations) tomberont de plein droit au fur et à mesure de la libération du territoire28.

Partout, les chefs de corps interrogés résistent. Se sentant menacées, les autorités judiciaires font savoir aux autorités allemandes qu'elles ne reconnaissent aucun pouvoir de nomination au pouvoir occupant. Pour consolider cette position, le procureur général Terlinden rédige un argumentaire $\underline{29}$. L'argument central est tiré du droit international : l'occupant, qui ne dispose que d'un pouvoir de fait par nature provisoire, n’a pas la capacité de nommer à vie des magistrats inamovibles $\underline{30}$.

La question des nominations, au-delà des aspects patriotiques qu'elle comporte, touche aussi au statut personnel de la magistrature : «[...] en faisant siéger des magistrats ayant prêté serment avec des personnes désignées par l'occupant et n'ayant pas prêté serment, on porterait atteinte aux règles essentielles de leur institution, on toucherait au statut personnel de la magistrature belge et on violerait son indépendance $\gg \underline{31}$.

Pour l'univers fermé des magistrats belges, la menace est autant professionnelle que politique. L'arrivée de juges activistes au mépris des usages en matière de nomination est perçue comme une véritable intrusion. Aussi la note Terlinden contient-elle une mise en garde à l'autorité allemande. Des nominations auront des conséquences funestes pour le fonctionnement de la justice. Les magistrats légaux devront considérer les nominations comme inexistantes et les décisions des personnes nommées comme dépourvues de toute autorité et de toute valeur. "Quel trouble et quelle désorganisation dans la vie publique ! $\$ \underline{32}$.

Aux dires du procureur général, cette opposition stoppe pendant quelques mois les nominations dans la magistrature et le notariat33. Mais, au tournant de 1917-1918, soit peu après la proclamation d'autonomie des Flandres, la digue cède. Plusieurs nominations sont promulguées, toutes issues du vivier activiste flamand. Fin janvier 1918, les trois premiers juges de paix activistes prennent leurs fonctions.

Dans ce processus, les Allemands semblent jouer un rôle secondaire, se bornant à satisfaire les prétentions activistes. Les archives du Conseil des Flandres (Raad van Vlaanderen - RVV) $\underline{34}$ révèlent que certains membres suspectent les autorités occupantes de retarder intentionnellement les nominations $\underline{35}$.

De son côté, le chef de l'administration civile pour la Flandre, Alexander Schaible, incrimine le faible nombre decandidatures valables. Ainsi, fin janvier 1918, le RVV n'a pu proposer que quatre candidats pour des places de juges de paix parmi lesquels trois seulement ont été nommés $\underline{36}$.

En Wallonie, la situation est tout autre. Le chef de l'administration civile allemande, Edgar Haniel, qui ne subit pas la pression des activistes, partage, au contraire de Schaible, les réticences des magistrats. Comme le mentionne son rapport semestriel de juin 1917 à janvier 1918, « un intérêt particulier en ce domaine n'existe pas [...] tant que les tribunaux fonctionnent de manière régulière», surtout parce que de telles nominations - si même l'on trouvait déjà des personnes qui conviennent - pourraient avoir des conséquences très indésirables $\underline{37}$.

Les nominations de magistrats activistes forment une première salve importante contre l'organisation 
judiciaire. La réponse du corps judiciaire marque elle aussi une rupture avec la prudence antérieure. Comme annoncé par le procureur général Terlinden, les magistrats fraîchement nommés par l'occupant font l'objet de mesures vexatoires de la part des magistrats en place. Le nouveau juge de police à Anvers, Henry Quakkelaer, par exemple, voit son travail systématiquement contrecarré par le parquet qui fait appel de toutes ses décisions $\underline{38}$.

Fin 1917, la Flamenpolitik offre une nouvelle cause de rupture. La proclamation de l'indépendance des Flandres par les activistes provoque en territoire occupé et dans la diaspora belge une vague d'indignation. Forte de celle-ci et aiguillonnée par des politiciens, la Cour d'appel de Bruxelles enjoint, le 7 février 1918, à son procureur général de poursuivre les leaders du mouvement. Deux d'entre eux sont arrêtés... et presque immédiatement remis en liberté par le pouvoir occupant qui punit les conseillers de la cour pour «mouvement politique»: tous sont suspendus et trois présidents sont déportés en Allemagne. Devant cette violation flagrante de l'indépendance de la justice, la Cour de cassation suspend solennellement ses fonctions, bientôt suivie par de nombreuses juridictions belges. La grève judiciaire se prolongera jusqu'à l'Armistice, forçant les Allemands à mettre en place leurs propres tribunaux $\underline{39}$.

La question des nominations, surgie très peu de temps avant la grève, a très probablement pesé dans la décision de maintien de celle-ci : à quoi bon rejoindre son siège si l'édifice est gangréné ?

Pendant les années 1930, alors que l'hypothèse d'une seconde occupation du territoire est sérieusement envisagée, le procureur général honoraire près la Cour d'appel de Bruxelles, Jean Servais, suggèrera, en cas d'invasion, de déléguer aux procureurs généraux le pouvoir de nomination $\underline{40}$.

\section{0-1945}

Durant la Seconde Guerre mondiale, la Personalpolitik de l'occupant est plus ambitieuse. Elle vise non seulement à placer des magistrats flamands et surtout des magistrats favorables à l'Ordre nouveau mais aussi à briser la résistance des magistrats belges. L'autorité allemande agit alors indirectement par le biais des secrétaires généraux successifs du ministère de la Justice. En effet, la nomination des magistrats est, en 1940-1944, une prérogative du secrétaire général de la Justice.

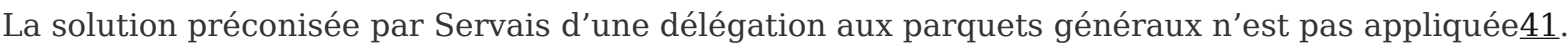

Les chefs de corps continuent cependant de jouer un rôle important. Le secrétaire général demande leur avis et respecte celui-ci dans la plupart des cas. Par ailleurs, les techniques de délégation, de suppléance et de prolongation automatique des mandats mises en œuvre pendant la première occupation sont appliquées le plus largement possible pour éviter le contrôle de l'occupant.

Néanmoins, par le biais du Gruppe Justiz, l'administration allemande contrôle les nominations effectuées et s'immisce régulièrement dans le processus en exerçant une pression variable selon le moment et le contexte sur le secrétaire général de la Justice.

En matière de nominations judiciaires, les desseins allemands ne sont pas homogènes. De la même façon que l'administration civile est poussée par les activistes à s'ingérer dans les nominations de magistrats belges en 1917, les responsables de l'administration militaire sont influencés par les intérêts des groupements belges d'Ordre Nouveau. Différentes mesures sont prises par les Allemands - cédant souvent aux demandes faites en ce sens par les partis collaborationnistes pour mieux contrôler les nominations. Cette ingérence augmente avec le temps. 
Magistrats et occupants, quelques jalons d'une comparaison diachronique (1914...

La première des mesures allemandes est une ordonnance du 4 avril 1941qui dispose que la nomination des chefs de corps de la magistrature nécessite l'accord de la Militärverwaltung 42 . Selon des sources allemandes, cette ordonnance aurait notamment servi à écarter des candidats réputés germanophobes et à privilégier des candidats flamands. Du point de vue de l'administration militaire, en effet, « les revendications flamandes étaient justifiées dans la mesure où les Flamands étaient sous-représentés dans la magistrature par rapport à leur proportion de la population totale. Les places dirigeantes, notamment, étaient presque exclusivement occupées par des Wallons même en zone flamande $» \underline{4} \underline{3}$.

Les groupements d'Ordre Nouveau - notamment le VNV et les Rexistes - se montrent insatisfaits de la restriction de la tutelle allemande aux places dirigeantes et pressent la Militärverwaltung d'obtenir davantage de contrôle et un changement plus politique et palpable du visage de la magistrature, en considérant que «les nominations sont le terrain par excellence sur lequel la résistance des tribunaux peut être combattue et brisée $» \underline{4}$.

$\mathrm{Au}$ début de l'occupation, le secrétaire général Ernst de Bunswyck $\underline{45}$ doute de la légalité des arrêtés des secrétaires généraux. Par prudence, il s'abstient de procéder à des nominations. Pour pallier l'absence de magistrats ou de suppléants provoquée par les abandons de poste et par les mobilisations notamment au niveau des justices de paix et des tribunaux de première instance, il a recours à l'arrêté du 22 juillet 1940 et à l'arrêté du 20 décembre 1940, qui permettent de désigner temporairement des avocats comme suppléants $\underline{46}$.

$\mathrm{Au}$ printemps 1941, après l'interdiction d'exercice prononcée contre lui suite à l'ordonnance concernant le vieillissement des cadres (voir infra), un nouveau secrétaire général entre en fonction : Gaston Schuind, conseiller près la Cour d'appel de Bruxelles. L'administration militaire lui fait comprendre qu'elle attend de lui qu'il veille à corriger par ses nominations la sousreprésentation des magistrats flamands. De fait, avec l'arrivée de Schuind au secrétariat général, on observe une nette augmentation des nominations. La phase de prudence du début est révolue. Un pic de nominations dans la magistrature a lieu lors de la rentrée judiciaire de 1941.

Par ailleurs, suite à une demande de l'administration militaire, Schuind nomme, à l'été 1941, l'avocat furnois Selschotter, comme chef de son cabinet47. L'appel du ministère de la Justice à un juriste flamand à ce poste-clé vise stratégiquement à apaiser l'occupant. Les mouvements d'Ordre Nouveau se plaindront plus tard que l'intégration de Selschotter au ministère de la Justice n'a servi à rien, les nominations restant «anti-flamandes et germanophobes » 48 . De fait, le profil de magistrat souhaité par les partis tel que le VNV, c'est-à-dire politisé, ouvertement national-flamand, germanophile et docile, est pratiquement inexistant chez les juristes.

L'occupant doit ménager ses alliés et cède de temps à autre aux demandes inlassables de personnalités de l'Ordre Nouveau d'appuyer certains candidats ou d'en destituer d'autres. Les crises judiciaires (été 1942 et hiver 1942/1943) $\underline{49}$ mettent de l'huile sur le feu et les autorités allemandes se demandent s'il ne serait pas judicieux d'élargir la tutelle sur les nominations à toutesles fonctions de magistrat. Ces réflexions coïncident avec l'édiction d'arrêtés-lois par les secrétaires généraux qui visent à modifier le paysage politique belge. Ces arrêtés-lois, créant de grandes agglomérations urbaines afin de les substituer à la multitude de communes qui composent les grandes villes belges, leur ont été dictés par la Militärverwaltung. Pourtant, au début de l'occupation, il y avait un large consensus parmi les secrétaires généraux sur l'illégalité des modifications de la structure politique du pays. Conscients que, dans ce contexte tendu, une résistance de la part des juges, 
ayant le droit de se prononcer sur la légalité des arrêtés-lois, est à prévoir, les autorités allemandes semblent s'inquiéter plus qu'auparavant de la question des nominations dans la magistrature. En même temps, dans les cercles de l'Ordre Nouveau, la politique de nomination du secrétaire général Schuind est de plus en plus décriée $\underline{50 .}$

Pourtant, les aspirations des groupements d'Ordre Nouveau en matière de nomination sont malaisées à réaliser. Un problème de défaut de qualification des candidats proposés par les partis collaborationnistes se pose, ce dont le Gruppe Justiz est pleinement conscient $\underline{51}$. Néanmoins, pour éviter des nominations qui vexeraient les mouvements d'Ordre Nouveau et pour donner l'impression qu'on veille à respecter leurs souhaits, la Militärverwaltung demande officieusementau secrétaire général de la Justice de la consulter au plus tard deux semaines avant chaque nomination de magistrat $\underline{52}$.

Ce système se heurte au fait que l'administration militaire ne dispose pas d'assez d'effectifs pour effectuer une enquête sur les candidats. Dès lors, le chef du Gruppe Justiz van Randenborgh se prononce clairement contre l'extension de l'ordonnance du 4 avril 1941. Van Randenborgh considère que le processus d'information sur les candidats est compliqué et nullement infaillible. Il objecte aussi que si la Militärverwaltung a officiellement donné le feu vert pour une nomination, celle-ci sera d'autant plus difficile à annuler et que, somme toute, des candidats flamands ne sont pas disponibles en nombre suffisant $\underline{53}$.

La nomination d'un procureur du Roi à Hasselt, notamment, provoque un revirement de la politique de nominations du secrétaire général Schuind. En septembre 1941, le procureur du Roi de Hasselt, Massa, est interdit de fonctions. La mesure est uniquement motivée par des attaques contre Massa du camp collaborationniste local. Selon les traditions, le secrétaire général projette de nommer le substitut Vandevelde qui remplit déjà temporairement ce poste en remplacement de Massa. Le Gruppe Justiz y est également favorable. Mais le VNV s'oppose vivement au candidat qu'elle juge opportuniste $\underline{54}$. Finalement, on désigne le substitut du procureur du Roi de complément du tribunal d'Anvers, Rubens. Ce dernier est accepté par les groupements flamands d'Ordre Nouveau et l'administration allemande donne son accord $\underline{55}$. Ici, l'occupant a donc largement interféré dans le domaine des nominations de magistrats, une prérogative normalement réservée au secrétaire général.

Pour éviter que cette situation ne se reproduise dans d'autres parquets et notamment à Bruxelles, Anvers et Louvain, où les places de procureur sont vacantes, le secrétaire général Schuind s'abstient de procéder à de nouvelles nominations $\underline{56}$. Mais cette stratégie d'évitement est rapidement détectée par les Allemands $\underline{57}$.

Entretemps, les responsables du Gruppe Justiz s'étant rendu compte de l'impossibilité de procéder à une enquête correcte sur l'attitude politique du magistrat candidat en deux semaines, la demande d'inspection préalable est retirée (vers février 1943). De plus, au sein du Gruppe Justiz, on réalise que les magistrats nommés sur proposition du VNV ne sont pas nécessairement plus dociles. Le cas du procureur du Roi d'Anvers, Edouard Baers, fortement appuyé par le VNV pour accéder à ce poste en février 1942, est très éclairant. Au début de l'année 1943, la Militärverwaltung constate avec le plus grand étonnement que ce procureur se prononce fermement contre la collaboration de la police belge aux mesures allemandes. Pour cette raison, l'occupant lui interdira ensuite l'exercice de sa fonction $\underline{58}$. Le comportement de Baers est une sévère déconvenue pour les collaborateurs $\underline{59}$. 
Magistrats et occupants, quelques jalons d'une comparaison diachronique (1914...

Malgré la critique véhémente des partis d'Ordre Nouveau contre la politique des nominations de Gaston Schuind, le Gruppe Justiz soutiendra longtemps le secrétaire général. Van Randenborgh le défend encore au moment où les voix en faveur de sa destitution se font de plus en plus fortes 60 . Le 17 septembre 1943, Schuind est destitué pour diverses raisons. On désigne l'ancien administrateur de la Sûreté publique, Robert de Foy, comme secrétaire général faisant fonctions, un choix pour le moins étonnant puisque de Foy avait été détenu par les Allemands au début de l'occupation en raison de son implication dans les arrestations de mai 1940.

En novembre 1943, avec l'arrivée au poste de de Foy, la question de l'extension de l'ordonnance du 4 avril 1941 à l'ensemble des nominations revient sur la table. L'initiative vient maintenant du chef de l'administration de la Militärverwaltung von Craushaar. De Foy invoque des affaires plus urgentes à régler lors de son entrée au secrétariat général et parvient, dans un premier temps, à différer toute intervention.

Pendant trois ans, les secrétaires généraux successifs réussissent tant bien que mal à éviter l'ingérence totale des Allemands dans les nominations de magistrats. Mais, en mars 1944, la direction de la Militärverwaltung estime qu'il est maintenant temps de faire dépendre chaque nomination de son accord et publie à cette fin l'ordonnance du 16 mars 19441․ Cette décision intervient dans le contexte de radicalisation qui caractérise les derniers mois de l'occupation en Belgique. L'occupant a alors cruellement besoin d'une justice belge docile. Or celle-ci manifeste une hostilité de plus en plus marquée à l'occupant et à l'Ordre Nouveau62. Par un mouvement de balancier, les nominations se font rares. Les chefs de corps ont massivement recours à des délégations temporaires.

En définitive, les nominations des secrétaires généraux furent extrêmement nombreuses, avec au moins 250 dossiers de magistrats qui contiennent un arrêté de nomination datant de la période de l'occupation 63 .

\section{Une seconde catégorie d'atteintes à l'indépendance de la magistrature : les mesures de rigueur à l'égard de magistrats}

\section{4-1918}

\section{Quelques chiffres}

De la période qui suit l'invasion de l'été 1914 à la grève des magistrats en février 1918도, quelques nonante incidents ont été relevés dans les rapports d'après-guerre65, impliquant une soixantaine de magistrats. Ce chiffre globalise les immixtions de l'occupant dans le cours de la justice et les mesures dirigées contre des magistrats, de la simple intimidation à la déportation. Il est certainement en deçà de la réalité pour ce qui concerne les obstacles mineurs posés à l'exercice de la justice.

Pour la moitié des magistrats, il s'est agi de simples pressions ou de mesures d'intimidation (menaces verbales, convocations et interrogatoires à la Kommandantur). Onze ont été privés de liberté, qu'ils soient détenus sans jugement ou emprisonnés à la suite d'une condamnation (quelques cas seulement). Vingt magistrats ont été condamnés, à des peines de prison ou, majoritairement, d’amende.En général, les détentions sans jugement ne durent que quelques jours, une dizaine au maximum. Les condamnations à des peines de prison ont surtout frappé l'action clandestine 
(quatre cas) $\underline{66}$ - de quelques mois de détention pour vente de journaux prohibés à plusieurs années accompagnées de travaux forcés dans les cas plus lourds, pour les crimes de «trahison » (exfiltration de soldats, renseignement). Pour des actes commis dans l'exercice de leurs fonctions, un magistrat a subi une détention de plusieurs mois dans une prison belge et trois magistrats ont été déportés en Allemagne. Ces déportations ne sont pas des sanctions pénales mais des mesures d'écartement arbitraires, décidées par le pouvoir militaire, sans jugement et souvent sans que le motif en soit exprimé. Enfin, trois magistrats ont été interdits de fonctions par le gouverneur général allemand à titre disciplinaire.

Aucun magistrat n’a été exécuté. Un juge de paix déporté en 1916 est cependant décédé de mort naturelle (des suites de ses conditions de détention ?) quelques mois plus tard, au camp allemand où il était détenu pour avoir poursuivi ses fonctions sans avoir signé la déclaration de loyauté exigée par l'autorité occupante.

\section{Typologie des incidents et qualité des magistrats visés}

Si des comportements individuels comme l'engagement clandestin au service des alliés ont pu jouer un rôle, c'est d'abord la compétence des magistrats concernés qui explique l'intervention allemande.

L'intérêt des occupants pour certaines des compétences des juges de paix est ainsi à l'origine d'immixtions. Les incidents rapportés concernent au premier chef des affaires locatives 67 , au second des levées autoritaires de scellés, qui permettent à l'occupant de réquisitionner matériel et locaux et, enfin, en matière de police, des infractions aux règlements communaux (bals, cabarets).

Les juges d'instruction et les membres des parquets sont également soumis à des pressions. Leur capacité d'enquête et le rôle essentiel qu'ils jouent dans les poursuites judiciaires en font deux catégories de magistrats potentiellement gênants pour l'occupant. L'exécution des peines, prérogative du parquet, est aussi source de conflits. L'occupant, en mal de forces vives, puise régulièrement dans le réservoir des détenus des recrues allemandes pour son armée ou des natifs prêts à seconder les services de contre-espionnage allemands $\underline{68}$. Sont aussi malmenés les juges des enfants qui, dotés de compétences larges, interviennent au niveau de l'instruction, du jugement et l'exécution des mesures. Des mineures prostituées qui entretiennent des relations avec des Allemands, par exemple, et qui font l'objet de mesures de placement en institution par des juges des enfants, sont ainsi à plusieurs reprises remises en liberté par l'occupant $\underline{69}$.

Par contre, les autres magistrats du siège sont très peu atteints. À la différence des juges de paix, les magistrats de première instance sont rarement pris à partie pour la jurisprudence rendue. L'administration civile, dans ses rapports officiels, se glorifie d'ailleurs de respecter l'indépendance des juges « au point d'admettre que des tribunaux déclarent illégales des ordonnances du gouverneur général » $\underline{70}$.

Quant à la haute magistrature, siège et parquet, elle est quasiment sortie indemne des trois premières années d'occupationz1.

$\mathrm{Au}$ niveau local, la situation est très contrastée. Dans le ressort de Gand qui correspond à la principale zone d'étape des armées allemandes et, en particulier, dans la zone des opérations, à l'arrière immédiat du front, le régime essentiellement militaire frappe de façon beaucoup plus arbitraire. Le pouvoir judiciaire belge est aussi moins respecté, ce qui se marque statistiquement 
Magistrats et occupants, quelques jalons d'une comparaison diachronique (1914...

par un taux d'immixtion proportionnellement plus important que dans les deux autres ressorts. Même les hauts magistrats ne semblent pas protégés par leur statut. Le procureur général Alexis Callier, par exemple, est pris deux fois en otage et on lui refuse systématiquement tout passeport pour ses déplacements professionnels. Il est même condamné en octobre 1916 à une amende de 2000 marks à titre disciplinaire pour avoir transmis aux procureurs du ressort un arrêt de la Cour de cassation qui déplaît aux autorités d'occupation72. C'est, avant la grève de février 1918, la seule démarche punitive ciblant un haut magistrat belge.

Dans la zone d'étape des armées allemandes, la marge de négociation du pouvoir judiciaire paraît faible. La situation est pire encore dans la zone des opérations. Plusieurs mesures iniques y frappent des magistrats inférieurs. Le juge de paix d'Hooghlede, par exemple, est suspendu de ses fonctions pour avoir quitté sa résidence alors que la zone, en plein front, est devenue inhabitable.

De façon générale, l'indépendance des magistrats nationaux est malmenée lorsque des protégés du régime d'occupation sont poursuivis par la justice belge. Il peut s'agir de collaborateurs (activistes, rédacteurs de la presse censurée, trafiquants...), de civils ou d'entreprises d'origine allemande ou même de simples particuliers belges dont on veut gagner les faveurs. Quand c'est possible, la stratégie la plus simple est de rattacher ces individus à l'armée allemande pour qu'ils relèvent de sa justice militaire.

À côté des dessaisissements formels, d'autres sont déguisés. Un procédé d'écartement des magistrats belges de certaines affaires consiste à pénaliser, lorsque la situation s'y prête, leurattitude 'germanophobe'. L'arrêté du 4 septembre 1915 est ainsi le principal instrument juridique de protection des alliés de l'occupant73. Il punit et confie aux tribunaux militaires allemands tous ceux qui essayent de nuire ou de dissuader par des menaces les personnes manifestant des sentiments germanophiles. Extrêmement vague, l'arrêté se prête à une interprétation large.

Un second procédé consiste à invoquer que les arrestations n'ont rien à voir avec l'exercice des fonctions judiciaires et qu'elles répondent à un motif d'ordre militaire. La simple invocation de raisons militaires coupe l'herbe sous le pied à des autorités judiciaires belges particulièrement soucieuses de ne pas commettre d'ingérence dans le domaine des autres pouvoirs.

\section{Réactions de la haute magistrature - La solidarité corporative à l'épreuve}

La plupart du temps, les violations de l'indépendance des magistrats et les éventuelles pressions dont ils sont victimes font l'objet de rapports et de protestations du parquet adressées aux autorités allemandes. Dans les cas les plus graves, la plus haute magistrature du pays - la Cour de cassation - est saisie par son parquet de la question de l'opportunité d'une protestation officielle.

$\mathrm{Au}$ sein de la magistrature, le soutien aux magistrats poursuivis prête systématiquement à discussion. Les délibérations des conseillers de cassation révèlent des failles dans la solidarité corporative qu'on suppose toujours si puissante dans la magistrature.

Le sort de trois magistrats frappés dans l'exercice de leurs fonctions a fait l'objet de délibérations de la Cour de cassation. Dans un seul cas, celle-ci a protesté officiellement et réclamé le retour du magistrat déporté.Elle n'a pas souhaité intervenir pour les deux autres. La différence de traitement renvoie probablement à une conception déontologique de l'action du magistrat en temps de guerre, peu éloignée sans doute de celle du temps de paix. En effet, le magistrat secouru est un magistrat modèle. Il applique la loi avec impartialité. Au contraire, les deux autres ont 
donné à leur intervention judiciaire un caractère politique et leurs propos manquent de mesure ; ils sont imprudents. Leur témérité met en danger le pouvoir judiciaire tout entier. La neutralité, vertu ordinaire de la magistrature, revêt ainsi, dans le contexte de l'Occupation, une dimension stratégique.

La grève de 1918, initiée par la Cour de cassation en réaction aux mesures disciplinaires infligées aux conseillers d'appel bruxellois, rompt avec l'attitude attentiste observée jusque-là. Le fait que l'occupant ait, pour la première fois, sanctionné des hauts magistrats, explique probablement en partie ce revirement.

\section{0-1945}

Durant la seconde occupation, la situation des magistrats est plus défavorable encore. Différents procédés sont utilisés par l'occupant pour écarter (temporairement ou définitivement) les éléments indésirables.

\section{Les interdictions d'exercice sur la base d'ordonnances du Militärbefehlshaber}

Un premier type de mesures d'écartement est prise par voie d'ordonnances allemandes. Dans le cas d'une interdiction d'exercice, le commandant militaire donne l'ordre au secrétaire général de la Justice de destituer le magistrat74. En ce qui concerne la magistrature, les interdictions d'exercice sont motivées par les ordonnances suivantes.

\section{Ordonnances relatives à l'exercice d'une activité publique}

Le 18 juillet 1940, l'administration militaire promulgue une ordonnance qui interdit la rentrée au pays des membres de l'ancien gouvernement Pierlot75. Les personnes qui détiennent une fonction publique dirigeante et qui ont fui la Belgique lors de l'invasion ne sont autorisées à reprendre une activité publique que sur autorisation du commandant militaire. Les magistrats, dans leur ensemble, sont concernés : "Une activité publique dans le sens de cette ordonnance est exercée par quiconque occupe une situation officielle ou publique ?...? Le Commandant militaire décidera dans les cas douteux si les conditions prévues au § 2 sont remplies »76.La disposition décisive se trouve à l'alinéa 2 du paragraphe 3 : «Il se réserve, en outre, le droit d'interdire également l'exercice d'une activité publique à certaines personnes qui ne tomberaient pas sous l'application des cas prévus par cette ordonnance $\gg \underline{77}$.

Cet instrument juridique va bien plus loin que l'ordonnance sur la germanophobie de septembre 1915. Par cette possibilité d'interdire le droit d'exercer à tout titulaire d'une fonction publique, les Allemands s'octroient un pouvoir étendu sur l'ensemble de l'appareil administratif et judiciaire belge. L'interdiction de fonctions sera fréquemment utilisée par les Allemands pour exclure des personnes politiquement indésirables des institutions belges. Le 5 août 1940, les catégories de fonctions visées par l'ordonnance sont précisées : pour la magistrature, il s'agit des chefs de corps.

Le 19 décembre 1940, une ordonnance complétant celle du 18 juillet est publiéeㄹ. L'alinéa 5 stipule que «toute nomination aux fonctions et emplois des personnes visées aux alinéas 1 à 4 est soumise à l'approbation préalable du Commandant Militaire»79. L'administration militaire se réserve donc le droit d'influencer directement les nominations de remplaçants de personnes 
Magistrats et occupants, quelques jalons d'une comparaison diachronique (1914...

évincées par l'ordonnance du 18 juillet.

Le 20 novembre 1942, une deuxième ordonnance complétant l'ordonnance du 18 juillet 1940 est publiée. Elle étend cette fois le cercle de personnes visées à toutes les fonctions publiques et non plus comme auparavant aux seules positions dirigeantes. Elle précise entre autres que l'application de l'ordonnance allemande n'empêche pas des sanctions disciplinaires plus graves $\underline{80}$. Un autre changement déterminant pour les personnes écartées est opéré à ce moment, par ordre donné au secrétaire général de la Justice : tandis que les personnes destituées bénéficiaient, selon le droit belge, d'une indemnité la première année suivant la mise à disposition et ensuite d'une pension, les Allemands décident que les indemnités pleines ne seront plus payées au-delà de trois mois $\underline{81}$.

Ces dispositions plus sévères surgissent dans un contexte de radicalisation politique. L'administration militaire constate que les mouvements de Résistance recrutent de plus en plus parmi les fonctionnaires et autres détenteurs d'une fonction publique. Elle réagit (entre autres) par une intensification des interdictions d'exercice 82 .

Dans l'ensemble, cependant, la Militärverwaltung applique l'ordonnance avec modération. Selon la ligne suivie par le commandant militaire en matière d'interdictions d'exercice, celles-ci ne sont «à appliquer que quand les intérêts de la puissance occupante sont heurtés et quand d'autres moyens ?...? ne peuvent plus être envisagés. Une interdiction d'exercice ne doit pas mener à une situation dans laquelle des officiers d'une fonction publique dérangeante, qui sont efficaces dans leur métier et qui conviennent aux devoirs dans le cadre de la loi, soient destitués seulement pour éviter des difficultés, alors qu'un comportement d'opposition et malintentionné ne peut pas leur être reproché. En fin de compte, après une destitution, la question du remplacement doit encore être examinée $\gg \mathbf{8 3}$.

L'ordonnance a été utilisée pour écarter environ (évaluation minimale) vingt à trente magistrats à différents moments de l'occupation et pour des motifs divers, alors que ceux-ci n'avaient, pour la plupart, pas quitté la Belgique en mai 1940.

L'occupant ne se prononce jamais sur les motifs des interdictions d'exercice, pour ne pas donner l'occasion à la magistrature et au secrétaire général de la Justice de protester contre les violations de l'indépendance de la magistrature. Il existe une exception. Le vice-président du tribunal de première instance de Gand, Karel Reychler, est interdit de fonctions en date du 29 septembre 1941, après avoir rendu un jugement contraire aux intérêts allemands. Les représentants de l'Oberfeldkommandantur font l'erreur d'expliquer longuement leur décision par écrit. Ceci permit à la Cour de cassation de démontrer l'illégalité de cette décision, puisqu'elle heurte l'indépendance de la magistrature, et d'obtenir la réinstallation de Reychler dans sa fonction. Suite à cet événement, l'administration militaire s'abstint systématiquement de faire connaître les motifs d'une interdiction d'exercice.

\section{Ordonnance sur le « rajeunissement des cadres»}

Le 7 mars 1941, l'administration militaire promulgue une « ordonnance contre le vieillissement des cadres de l'administration publique en Belgique $» \underline{84}$. Selon cette ordonnance, tous les titulaires d'une fonction publique cessent leurs fonctions lorsqu'ils atteignent l'âge de soixante ans. Le commandant militaire se réserve le droit d'autoriser des exceptions. Les magistrats du ministère public sont concernés par l'ordonnance. 
Notons qu'au sein de l'administration militaire, on n'a nullement songé aux magistrats lors de l'élaboration du texte. Sa promulgation vise surtout les secrétaires généraux indociles. Il se trouve en effet que les secrétaires généraux les moins dociles vis-à-vis de l'occupant - ceux de la Justice et des Affaires intérieures - ont plus de soixante ans. On y voit surtout un moyen de faire nommer Gerard Romsée à l'une de deux fonctions $\underline{85}$.

Comme en matière de nomination, les mouvements d'Ordre Nouveau se disent insatisfaits de l'ordonnance et critiquent surtout le fait que les magistrats du siège ne sont pas concernés $\underline{86}$. $\mathrm{Au}$ sein de l'administration militaire, on est conscient qu'un élargissement de l'ordonnance à la magistrature assise équivaudrait à la « désaffectation des cours et tribunaux, puisqu'environ un tiers des juges a dépassé les 60 ans et parce que la question de la relève est particulièrement compliquée ici »87. Dans un autre document, on peut lire que « tant que l'attitude de la magistrature du siège ne l'exige pas, une promulgation qui inclut les juges n'est pas à l'ordre du jour » $\underline{88}$. C'est donc en définitive moins pour des raisons légales (les magistrats du siège sont nommés à vie) que pour des raisons purement pratiques que la Militärverwaltung rejette l'extension de l'ordonnance aux magistrats du siège.

L'ordonnance livre cependant l'occasion aux Allemands de se débarrasser de quelques hauts magistrats du parquet89. Le VNV avait notamment souhaité un changement au parquet de la Cour d'appel de Gand et l'ordonnance offre l'occasion de le satisfaire $\underline{90}$. La procédure provoque une forte critique des secrétaires généraux par le gouvernement de Londres, pour avoir accepté de nommer des magistrats plus dociles aux places devenues vacantes par l'ordonnance du 18 mars 194191.

$\mathrm{Au}$ sein de l'administration militaire, on se félicite que l'ordonnance n'ait pas donné lieu à des manifestations de résistance au sein de l'administration de la Justice $\underline{92}$.

Selon les documents de l'administration militaire, quarante-quatre magistrats des différents parquets tombent sous l'application de l'ordonnance «vieillissement " $\underline{93}$. Ils indiquent aussi que des exceptions ont été faites dans la plupart des cas. En effet, les archives du ministère de la Justice ne comportent « que » une dizaine de dossiers concernant une interdiction d'exercice motivée par l'ordonnance du 18 mars $1941 \underline{\text { g4. }}$.

\section{Les arrestations de magistrats}

En plus de l'interdiction d'exercice, d'autres mesures de rigueur sont appliquées à des magistrats. Dans la plupart des cas, il s'agit d'emprisonnements pour motifs politiques.

Larrestation de magistrats est plus fréquente que l'interdiction d'exercice. Nous avons détecté une cinquantaine de cas de prisonniers politiques dans les dossiers des magistrats. Si nous y ajoutons les vingt à trente magistrats temporairement arrêtés comme otages, l'arrestation se profile comme la mesure de rigueur la plus appliquée lors de la seconde occupation. Il n'est pas rare d'ailleurs que le vécu d'un magistrat sous l'occupation soit marqué par la double expérience de l'interdiction d'exercice et de l'arrestation.

Si l'arrestation est un moyen de punir un magistrat individuellement, elle est aussi un moyen de dissuasion vis-à-vis de l'ensemble de la magistrature. Dans la plupart des cas, c'est l'activité (effective ou supposée) du magistrat dans un mouvement de résistance qui mène à l'arrestation. 
Magistrats et occupants, quelques jalons d'une comparaison diachronique (1914...

La plupart des magistrats arrêtés sont incarcérés en Belgique mais certains sont envoyés dans des camps de concentration en Allemagne. Une dizaine de magistrats décèdent durant ou à la suite de leur détention.

Du côté de l'administration militaire, aucun renseignement n'est donné sur les magistrats détenus. Les familles, les collègues ainsi que le secrétaire général de la Justice sont délibérément laissés dans l'ignorance.

En ce qui concerne les prises d'otages, les magistrats font partie d'une «catégorie » particulière : les otages de protection. Afin de protéger du matériel militaire ou prévenir des attaques des mouvements de résistance, les Allemands sélectionnent ces otages parmi les notables locaux. Notons que les magistrats choisis comme otages sont souvent ceux qui dérangent en raison de leur attitude (même si celle-ci reste dans le cadre de la légalité).

Après la Seconde Guerre mondiale, des critiques, provenant notamment de la gauche de l'échiquier politique, s'élèveront contre l'attitude adoptée par la magistrature sous l'Occupation. Les accusations les plus fréquentes incrimineront sa lâcheté et sa docilité à l'égard de l'administration militaire ainsi que son manque de combattivité pour défendre les individus considérés « subversifs » par le régime nazi.

Il est indéniable que la magistrature, en tant que groupe professionnel et haute autorité morale en territoire occupé, a échoué à protéger des groupes sociaux persécutés par le régime d'occupation. Il eût sans doute été possible, par exemple, d'invoquer plus souvent l'article 46 de la Convention de La Haye qui stipule que l'honneur et les droits des familles, le droit de vivre et de propriété privée des individus, ainsi que les croyances et pratiques religieuses doivent être respectées par

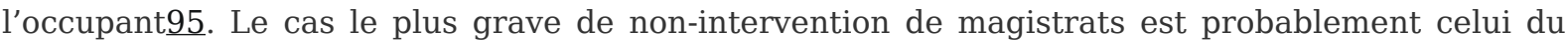
procureur du Roi d'Anvers Édouard Baers, qui n'a rien fait pour s'opposer aux rafles massives commises par la police anversoise contre la population juive de son arrondissement judiciaire en août et septembre 1942ㅁ․

Cependant, s'il est vrai que, en tant que groupe professionnel, la magistrature était loin de s'opposer systématiquement aux mesures allemandes, de nombreux magistrats participaient de manière individuelle et clandestine à des mouvements de résistance et à des services de renseignement.

Par ailleurs, les archives de l'administration militaire indiquent que le pouvoir judiciaire belge a su, par moments, mettre des bâtons dans les roues de l'occupant. Les crises judiciaires de 1942 et 1943 en forment un exempleg7. Ces crises se situent dans le contexte de l'opposition à diverses législations adoptées par les secrétaires généraux. L'institution d'une corporation agricole et de juridictions exceptionnelles en la matière, considérée illégale par certains juges, fut l'objet de la première crise. La légalité du pouvoir législatif des secrétaires généraux fut à nouveau, ensuite, mise en question par le pouvoir judiciaire lors du débat sur la formation de grandes agglomérations. Cette seconde crise a mené à une grève partielle de la magistrature bruxelloise à la mi-décembre 194298. Les deux crises se sont finalement soldées par des compromis.

D'autres exemples de résistance judiciaire concernent l'interprétation de la Convention de La Haye et les prérogatives respectives de la justice civile belge et de la justice militaire allemande. À citer aussi, la résistance de nombreux parquets contre la collaboration des services policiers belges à des mesures allemandes, comme par exemple la poursuite des réfractaires au travail obligatoire, ainsi que la poursuite pour port ou possession d'armes illégales, puisque le risque était grand 
d’identifier des résistants et de les voir ensuite transférés aux tribunaux militaires allemands $\underline{99}$.

Comme durant la plus grande partie de l'occupation de la Première Guerre mondiale (jusqu'à la grève de 1918), pour une majorité de magistrats, la priorité était de sauvegarder le pouvoir judiciaire sous l'Occupation. Continuer l'activité judiciaire, même au prix de compromis, était considéré comme la seule façon possible de contrer l'intrusion de représentants de l'Ordre Nouveau ou la mise en place d'une justice allemande, comme en 1918.

\section{Conclusions}

Les deux occupations allemandes, bien que caractérisées par de fréquentes violations de l'indépendance de la magistrature et des tentatives inédites, principalement pendant la Deuxième Guerre mondiale, de discipliner ses membres par un large éventail de mesures, eurent, du point de vue de la continuité du corps, un impact limité.

En effet, si les ingérences et mesures d'écartement obtenues par les partis collaborationnistes contre des magistrats formèrent la première menace professionnelle directe à laquelle étaient confrontés ceux-ci depuis 1830, ces mesures n'eurent qu'un effet provisoire. Celles prises par le pouvoir occupant en 1914-1918 et par les secrétaires généraux sous la pression allemande en 1940-1945 furent annulées lors du rétablissement des pouvoirs légitimes. Des mesures pénales et disciplinaires ont permis d'écarter après-guerre des avocats et magistrats séduits pendant l'occupation par une place ou une promotion dans la magistrature ou dans une institution mise en place par l'occupant $\underline{100}$.

Même pendant la seconde occupation qui vit la formation d'un minimum de 250 dossiers de nomination aux places vacantes, très peu parmi celles-ci ouvrirent la voie à une transformation sociologique du corps. Les profils de candidats élaborés par les collaborateurs belges des Allemands étaient très difficiles à trouver du fait notamment du conservatisme et de la neutralité politiques de la magistrature, un corps peu perméable aux idéologies nouvelles et extrêmes. Même les candidats les mieux étiquetés du point de vue des partis de l'Ordre nouveau, une fois nommés, rentrèrent dans les rangs. L'esprit de corps eut rapidement raison des singularités.

Les deux expériences d'occupation militaires, malgré une différence d'échelle impressionnante en termes d'atteintes à l'indépendance de la magistrature et un contexte politique différent, présentent d'importants parallélismes. On perçoit des deux côtés - magistrature (et secrétaires généraux le cas échéant) et occupants - une relative volonté de ménagement de l'adversaire. Négociations et tâtonnements sont la règle ; l'attaque frontale l'exception. Comme en 1914-1918, les Allemands responsables de la surveillance de la justice belge, pragmatiques, cherchent avant tout à garantir son fonctionnement, à éviter grèves et insurrections. Le spectre de la grève de 1918 joue certainement un rôle important même si ce rôle est rarement explicitement mentionné. La proximité professionnelle des membres de la Justiz Abteilung puis du Gruppe Justiz avec l'ordre judiciaire belge (ils sont eux-mêmes juristes, voire magistrats) favorise sans doute une certaine compréhension. Mais d'autres voix au sein de l'administration allemande, émanant de groupes davantage politisés, exigent une transformation plus radicale de la magistrature du pays occupé. Ces desseins contradictoires influencent la politique allemande et expliquent l'inconstance des pratiques.

4 D'après J. Gilissen, 30 magistrats (soit $10 \%$ du corps) ont été révoqués à l'automne 1830 dans le 
Magistrats et occupants, quelques jalons d'une comparaison diachronique (1914...

sillage de la révolution belge, pour leur proximité avec le gouvernement de Guillaume $\mathrm{I}^{\mathrm{er}}$ et le rôle joué dans les procès de presse intentés par ce dernier. Le phénomène paraît cependant modéré par rapport à l'épuration du parquet menée au même moment en France lors de l'installation de la Monarchie de Juillet (L'ordre judiciaire en Belgique au début de l'indépendance (1830-1832), dans Journal des Tribunaux, 1983, n 5266, p. 595). Quelques magistrats furent encore écartés en 1832 mais le gouvernement a renoncé à ce moment à mener une politique systématique d'épuration (J.-P. NANDRIN, Hommes et Normes. Le pouvoir judiciaire en Belgique aux premiers temps de l'indépendance (1832-1848), thèse de doctorat soutenue à l'UCL, 1995, p. 378-381).

11 Sur l'exercice de la justice militaire allemande en Belgique occupée, on connaît surtout la répression menée à l'égard des patriotes belges engagés dans des réseaux de renseignement : voir notamment J. VAN DER FRAENEN,Voor den kop geschoten : executies van Belgische spionnen door de Duitse bezetter (1914-1918), Roulers, 2009.

\section{Notes}

1 Cette publication a été réalisée dans le cadre du Pôle d'attraction interuniversitaire P7/22 «Justice \& Populations », Service public fédéral de programmation politique scientifique.

2 J. Logie, Les magistrats des cours et des tribunaux en Belgique (1794-1814) : essai d'approche politique et sociale, Genève, Droz, 1998.

$\underline{3}$ E. SCHANDEVYL, Changements ou continuité ? Rapports de force linguistique et (im)mobilité sociale dans la magistrature bruxelloise après la Deuxième Guerre mondiale, dans Les praticiens du droit du Moyen Age à l'époque contemporaine. Approches prosopographiques (Belgique, Canada, France, Italie, Prusse), éd. V. Bernaudeau, J.-P. NANDRin, B. Rochet, X. Rousseaux et A. TixHON, Rennes, Presses universitaires de Rennes, 2008, p. 255-270.

$\underline{5}$ À propos de l'impact de la Troisième République sur le recrutement social des magistrats, voir C. CHARLE, État et magistrats. Les origines d'une crise prolongée, dans Actes de la Recherche en Sciences sociales, XCVI, 1993, n 1, p. 39-48.

6 De façon générale, la comparaison entre les deux occupations allemandes de la Belgique est encore peu développée. Voir notamment : B. MAJERus, Occupations et logiques policières. La police bruxelloise en 1914-1918 et 1940-1945, Bruxelles, Académie Royale de Belgique, 2008 et P. NEFORS, " "Quand les fils de novembre nous reviennent en mai ..." "14-18" als gedragsbepalende factor tijdens de tweede Duitse bezetting », dans Quand les canons se taisent : actes du colloque international organisé par les Archives de l'État et le Musée royal de l'Armée et d'Histoire militaire (Bruxelles, 3-6 novembre 2008), éd. P.-A. TALLIER et P. NEFORS, Bruxelles, Archives générales du Royaume, 2010, p. 569-590.

7 Archives générales du Royaume (cité après AGR), Ministère de la Justice : Secrétariat général, $2^{\mathrm{e}}$ section et AGR, Ministère de la Justice, Secrétariat général, Service du personnel de la magistrature, Dossiers des magistrats, 1953-1980 (ci-après AGR, Dossiers des magistrats). Ont également été précieuses les archives 1914-1918 du parquet général de cassation toujours conservées au palais de justice de Bruxelles et les archives du premier président de la Cour de cassation, Joseph Jamar, conservées au CegeSoma.

8 Verwaltungsbericht des Verwaltungschefs, Belgien, 1914-1918 (Berlin, Geheimes Staatsarchiv 
Preußischer Kulturbesitz(cité après GSaPK)).

9 Fribourg en Brisgau, Bundesarchiv-Militärarchiv (ci-après BA-MA), fonds RW/36.

10 K. Peters, Les abandons de poste dans la magistrature lors de la Seconde Guerre mondiale, dans Cahiers d'histoire du temps présent (ci-après CHTP), n² 24, 2011, p. 163-186.

12 A. MeYers, La Magistrature et l'Occupation. Discours prononcé à l'audience solennelle de rentrée du $1^{\text {er }}$ octobre 1919 de la Cour d'appel de Liège, dans Journal des Tribunaux, 1919, col. 542.

13 L'introduction d'une administration civile en juillet 1944 n'implique pas de changements radicaux en raison de son caractère éphémère.

14 Proclamation à la population de la Belgique, dans Heeresgruppen-Verordnungsblatt für die besetzten Gebiete, n 1, 10 mai 1940, p. 2.

15 Notice destinée à toute la presse dans les territoires occupés de l'ouest, dans Ibidem, p. 16.

16 Conclusions et arrêt du 20 mai 1916, dans Pasicrisie, 1915-16, t. I, p. 375-418.

17 M. VAN DEN WIJNGAERT, Het beleid van het comité van de secretarissen-generaal in België tijdens de Duitse bezetting 1940-1944, Bruxelles, Palais des Académies, 1975, p. 29.

18 H. UMBreit, Les pouvoirs allemands en France et en Belgique, dans L'occupation en France et en Belgique 1940-1944, Actes du colloque de Lille, 26-28 avril 1985, éd. E. Dejonghe [extrait de Revue du Nord, 1987-1988, t. 1].

19 B. Majerus, La Sipo-SD en Belgique, Une police faible?, dans Vingtième Siècle, Revue d'Histoire, n 119, juillet-septembre 2013, p. 43.

20 G. Terlinden, Jubilé professionnel de Monsieur le Premier Président Du Pont, 14 septembre 1917, dans Bulletin de la Cour de cassation de Belgique, 1917, p. 13.

21 Au niveau cantonal, par exemple, la loi d'organisation judiciaire de 1869 (art. 8) prévoit « en cas d'empêchement légitime d'un juge de paix et de ses suppléants », le renvoi par le tribunal de première instance devant le juge de paix du canton le plus proche. L'article a notamment été invoqué à Louvain en l'absence prolongée du juge de paix de Faudeur et de ses deux suppléants, réfugiés en Angleterre. Le tribunal a renvoyé devant le juge de paix du second canton de Louvain l'ensemble des affaires du premier canton. Autre exemple : lorsqu'un juge d'instruction est momentanément empêché, les tribunaux, appliquant l'article 23 de la loi de 1869, désignent parmi leurs membres un juge titulaire pour le remplacer. Le procédé est accepté par l'autorité allemande, même dans l'étape.

22 Pour ne citer qu'eux, Eugène Dupont, premier président de la Cour de cassation, a septantehuit ans lors de la retraite des Allemands en 1918 ; Armand Hénoul, procureur général près la Cour d'appel de Liège, en a septante-six.

23 Exception révélatrice des enjeux de pouvoir et de souveraineté que revêt la question des nominations dans la magistrature : la demande de démission du conseiller près la Cour d'appel de Bruxelles, Charles Diercxsens, septante-deux ans, introduite en septembre 1914, est acceptée 
Magistrats et occupants, quelques jalons d'une comparaison diachronique (1914...

trois mois plus tard par le ministre de la Justice belge et par le gouverneur général allemand belge. Il ne sera cependant remplacé qu'en 1919.

24 Le décret de séparation administrative du pays en deux régions unilingues - la Flandre, dirigée de Bruxelles, et la Wallonie, de Namur - est une étape importante de la Flamenpolitik, politique allemande de faveur à l'égard de la communauté néerlandophone du pays. Il est à l'origine du dédoublement des ministères centraux à Bruxelles et Namur. Dans leur grande majorité, les fonctionnaires belges, refusant de se faire les complices de la politique allemande, cessent leurs fonctions et certains sont déportés. L'administration civile allemande est elle aussi dédoublée (P. Delforge, La Wallonie et la Première Guerre mondiale : pour une histoire de la séparation administrative, Namur, Institut Destrée, 2008).

$\underline{25}$ Qualificatif désignant la fraction minoritaire du Mouvement flamand qui, lors de la Première Guerre mondiale, choisit de collaborer avec l'occupant pour obtenir la réalisation de ses revendications linguistiques.

26 Verwaltungsbericht des Verwaltungschefs für Flandern für das Halbjahr August 1917 bis Januar 1918 (GSaPK, 32458, 474 vs-475 vs). Sur les relations très tendues entre magistrats et activistes, voir notamment la mercuriale de B. JOTTRAND, procureur général f.f. de Bruxelles pendant la guerre : Le pouvoir judiciaire et l'activisme séparatiste. Discours prononcé à l'audience solennelle [de la Cour d'appel de Bruxelles] du 4 décembre 1918, dans Journal des Tribunaux, 15 décembre 1918, $\mathrm{n}^{\circ} 2741$.

27 Flor Heuvelmans, membre important du Mouvement flamand à Anvers avant la guerre, est juge de police de cette ville lorsqu'il accepte de devenir secrétaire général du ministère flamand de la Justice.

28 Arrêté-loi du 8 avril 1917 déterminant l'effet des mesures prises par l'occupant et des dispositions prises par le gouvernement, dans Moniteur belge, 1917, p. 338-340.

29 Palais de justice de Bruxelles, Archives du parquet de la Cour de cassation, 2 (I). Celui-ci est officieusement inspiré par une étude de juillet 1917 cosignée par le conseiller d'appel et internationaliste Ernest Nys et par le conseiller de cassation liégeois Édouard Remy (E. NYS et E. REMY, La nomination de magistrats et de notaires par l'occupant, dans Belgique judiciaire, 1919, p. 5-10).

30 La doctrine vient en renfort de cette théorie. Terlinden cite le jurisconsulte allemand Franz de Holtzendorff, opposé à ce que l'occupant pourvoie lui-même à la nomination de juges (Éléments de droit international public, Paris, Rousseau, 1891, p. 179).

31 Palais de justice de Bruxelles, Archives du parquet de la Cour de cassation, boîte X, 2 (I).)(für den Monat März 1941 », in éral, s.d. ent.diale genre l's, par exemple, sont souvent autoritairemebnt lises en liberté

$\underline{32}$ Ibidem.

33 Note manuscrite du procureur général, s.d. (Palais de justice de Bruxelles, Archives du parquet de la Cour de cassation, boîte X, $n^{\circ} 2$ (I)).

34 Organe consultatif des activistes flamands auprès du pouvoir occupant. 
35 Ligue nationale pour l’Unité Belge, Les archives du Conseil de Flandre (Raad van Vlaanderen), Bruxelles, Dewarichet, 1928, p. 30.

36 Verwaltungsbericht des Verwaltungschefs für Flandern für das Halbjahr August 1917 bis Januar 1918 (GSaPK, 32458, 474 vs-475 vs).

37 Le rapport souligne la menace brandie par Terlinden à l'égard des juges nouvellement nommés (Verwaltungsbericht des Verwaltungschefs für Wallonien für das Halbjahr August 1917 bis Januar 1918. Haniel, 30. Januar 1918 (GSaPK, 32458, 30/391 verso)).

38 B. JotTRAND, Le pouvoir judiciaire et l'activisme séparatiste, p. 9.

39 Sur la grève de la magistrature : M. Bost et A. FRANÇOIS, La grève de la magistrature belge (février-novembre 1918). Un haut fait de la résistance nationale à l'épreuve des archives judiciaires, dans Histoire du Droit et de la Justice, une nouvelle génération de recherches, éd. D. Heirbaut, X. Rousseaux et A. WijfFels, Louvain-la-Neuve, Presses universitaires de Louvain, 2010, p. 19-43.

40 Documents de la Commission permanente de la mobilisation de la Nation (CEGESOMA, Archives Raoul Hayoit de Termicourt concernant le travail des institutions belges pendant l'occupation, $\mathrm{n}^{\circ} 2$ ).

41 Les archives n'expliquent pas pourquoi la solution préconisée par Servais n'est pas concrétisée en mai 1940. Cependant, le choix, au début de l'occupation, d'une délégation générale des pouvoirs ministériels vers les secrétaires généraux a éliminé cette option. Les Allemands entendent disposer d'un interlocuteur unique par département.

42 BA-MA, RW 36/397, p. 10. Le 19 mai 1941, une ordonnance allemande complémentaire à celle du 4 avril 1941 précise que l'ordonnance concerne aussi les remplaçants potentiels des chefs des parquets mais pas les suppléants des chefs de corps dans la magistrature assise.

$\underline{43}$ Idem, p. 11.

$\underline{44}$ « De benoemingen zijn het terrein bij uitstek waarop den tegenstand van het gerecht kan bevochten en gebroken worden » (« De benoemingen in het gerecht onder het beleid van den Heer Schuind », AGR, Dossiers des magistrats, dossier $n^{\circ} 2118$ ).

45 Ce secrétaire général était le chef de cabinet du ministre de la Justice Carton de Wiart pendant la Première Guerre mondiale.

46 «Lorsque les circonstances commandent de pourvoir d'urgence aux besoins du service d'un tribunal de première instance, le Président du Tribunal peut être autorisé par le Ministre de la Justice, sur avis du Premier Président de la Cour d'appel, à assumer temporairement des avocats inscrits au tableau de l'ordre pour exercer les fonctions de juge ?...? » (art. $1^{\text {er }}$ de l'arrêté du 20 décembre 1940, modifiant l'arrêté-loi du 6 novembre 1939 relatif au remplacement temporaire des magistrats rappelés sous les armes (cité dans AGR, Dossiers des magistrats, dossier de Louis Berger, $\left.n^{\circ} 115\right)$.

47 À cette fin, Selschotter venait d'être nommé par Schuind juge à Bruxelles. 
Magistrats et occupants, quelques jalons d'une comparaison diachronique (1914...

$\underline{48}$ «De benoemingen in het gerecht onder het beleid van den Heer Schuind » (AGR, Dossiers des magistrats, dossier $\left.n^{\circ} 2118\right)$.

49 Cf. par exemple C.-L. LouvEAUX, La magistrature dans la tourmente des années 1940-44, dans Revue de Droit Pénal et de Criminologie, 1981, passim ; et Idem, Le moindre mal, dans Juger, éd. F. RiNGelHeiM, n 6/7, Gilly, François Collinet, 1994, p. 28-30, ainsi que J.N.M.E. MICHIELSEN, The 'nazification' and 'denazification' of the courts of Belgium, Luxembourg and the Netherlands, Maastricht, 2004.

50 De benoemingen in het gerecht onder het beleid van den Heer Schuind, op. cit. : « À la lumière de la plus récente crise judiciaire on peut estimer quelle faute impardonnable a été commise ici. ?...? Dans la plupart des cas, d'un point de vue flamand et allemand, les nominations sont mauvaises».

51 K. PETERS, L'influence de l'administration militaire allemande sur les changements de personnel au sein de la magistrature belge (1940-1944), dans Histoire du droit et de la justice, op. cit., p. 64.

52 BA-MA, RW 36/397, p. 12.

53 BA-MA, RW36/407, p. 48.

54 Idem, p. 10 : «Le remplaçant actuel du procureur n'est pas jugé favorablement. Il n'est pas une personne réellement germanophobe et il travaille en général d’une manière tout à fait loyale, d'ailleurs il montre de la compréhension pour la situation actuelle, mais il est rejeté par le côté national-flamand. Il est notamment refusé par le V.N.V. ?...? pour son opportunisme, et ce surtout parce que des forces national-flamandes seraient soi-disant disponibles dans la province Limbourg ». Vandevelde sera nommé premier substitut au tribunal de première instance le 13 décembre 1942.

55 BA-MA, RW 36/384, p. 180.

56 De benoemingen..., op. cit.

$\underline{57}$ Cf. Tätigkeitsbericht des Chefs des Militärverwaltungsstabs, n²4, avril-juin 1943 »(BA-MA, RW 36/193, p. 138).

58 Tätigkeitsbericht der Gruppe Justiz für die Zeit vom 15. November 1942 bis 15. Februar 1943 (BA-MA, RW 36/382, p. 54). Plus tard, début 1944, Edouard Baers sera même accusé de participation à un service secret et détenu en Allemagne jusqu'en avril 1945.

$\underline{59}$ De benoemingen..., op. cit. : « De Heer Baers was voor 10 Mei 1940 vlaamsgezind doch is thans een bittere teleurstelling ».

60 Lettre du Gruppe Justiz, adressée au chef de l'administration de la Militärverwaltung, von Craushaar, le 23 mars 1943 (BA-MA, RW 36/422, p. 2) : «?...? Sa politique en matière de personnel judiciaire, qui fut souvent l'objet d'attaques violentes de la part du VNV et de Rex, a été trop souvent jugée unilatéralement en sa défaveur. 14 des 26 procureurs du Roi ont été destitués suite à nos mesures. La réoccupation de presque toutes ces places, en plus de plusieurs places de présidents de tribunaux de première instance et d'autres magistrats, notamment dans la zone 
flamande, a été faite par des personnalités proposées ou pour le moins tolérées par nous. Cela lui a d'ailleurs valu quelques affrontements avec la magistrature belge ».

61 Tätigkeitsbericht des Chefs des Militärverwaltungsstabs, n² 27, mars 1944 (BA-MA, RW 36/ 196, p. 32).

62 Niederschrift über eine Besprechung bei Herrn Ch.M.V. über Massnahmen auf dem Gebiet der Justiz ?...?, 7 mars 1944 (BA-MA, RW 36/402, p. 29).

63 Estimation minimale provisoire, sous réserve de l'achèvement de recherches en cours.

64 Les sanctions prises contre des magistrats à partir de la grève, évènement en rupture avec les trois années précédentes et qui marque une fuite en avant des protagonistes des deux bords, ne sont pas comptabilisées ici.

$\underline{65}$ Source : AGR, Commission d'enquête sur la violation des règles du droit des gens, des lois et des coutumes de la guerre.

66 Parallèlement à leurs fonctions judiciaires, un certain nombre de magistrats se sont engagés dans des activités clandestines, exposant dangereusement leur sécurité personnelle. À partir du dépouillement des dossiers des services patriotiques (AGR), Emmanuel Debruyne, spécialiste de la résistance, a dénombré huit magistrats effectifs engagés dans les réseaux de renseignement (communication personnelle).

67 Sur l'importance de la question locative en Belgique occupée, tant pour les autorités belges que pour l'occupant, voir : M. Bost, Le juge de paix et la question des loyers sous l'occupation allemande (1914-1918), dans Scènes uit de geschiedenis van het vredegerecht. Scènes de l'histoire de la justice de paix, éd. G. Martyn, Bruxelles, La Charte, 2011, p. 199-219.

68 M. Bost, X. Rousseaux et S. HoRvat, Les espions civils au service de l'ennemi, au prisme de la justice militaire belge. L'autre versant de la guerre de l'ombre (1914-1920), dans Journal of Belgian History, 2014, n² 2-3, p. 36-75.

69 Voir sur les conflits entre le juge des enfants bruxellois et l'administration allemande pendant la guerre : A. FRANÇOIS, Guerres et délinquance juvénile : un demi-siècle de pratiques judiciaires et institutionnelles envers des mineurs en difficulté (1912-1950), Bruxelles, La Charte, 2011.

70 Verwaltungsbericht des Verwaltungschefs von Sandt, August 1916-Januar 1917 (GSaPK, Rep. 89, 32457, 37-38.)

71 Cette situation change avec les poursuites enclenchées sur injonction de la Cour d'appel de Bruxelles contre les activistes en février 1918 qui entraine la déportation en Allemagne de trois présidents de chambre et la suspension des conseillers (voir M. BosT et A. FrANÇOIS, op. cit.).

$\underline{72}$ Il s'agit de l'arrêt de la Cour de cassation du 3 juillet 1916 affirmant la légalité de la création de gardes bourgeoises. Les autorités d'occupation de Gand ont imaginé que l'arrêt contenait un appel au soulèvement.

73 Arrêté du gouverneur général du 4 septembre 1915 concernant la répression des abus commis au préjudice des personnes germanophiles (C. HUBERICH et A. NICOL-SPEYER, Législation allemande pour le territoire belge occupé (textes officiels), La Haye, vol. IV, 1916, p. 303-304). 
Magistrats et occupants, quelques jalons d'une comparaison diachronique (1914...

74 Pour procéder à ces destitutions, le secrétaire général recourt à l'arrêté royal du 30 mars 1939 relatif à la mise en disponibilité des agents de l'État (Moniteur belge, 2 avril 1939, p. 2161).

75 Ordonnance du 18 juillet 1940 relative à l'exercice d'une activité publique en Belgique, dans Verordnungsblatt, $n^{\circ} 8,25 / 7 / 1940$.

76 Idem, alinéa 2 .

77 Selon l'historien Nico Wouters, la portée de cette disposition aurait initialement été sousestimée par les autorités belges (N. WouTERS, De Führerstaat : overheid en collaboratie in België (1940-1944), Tielt, 2006, p. 25).

78 Ordonnance du 19 décembre 1940, complétant l'ordonnance relative à l'exercice d'une activité publique en Belgique, dans Verordnungsblatt, $n^{\circ} 27,23 / 12 / 1940$, p. 8.

$\underline{79}$ Ibidem.

80 Deuxième ordonnance du 20 novembre 1942 complétant l'ordonnance relative à l'exercice d'une fonction publique en Belgique, dans Verordnungsblatt, n 90, 28 novembre 1942, p. 2.

81 Pourtant, les années précédentes, le commandant militaire estimait qu'il était préférable d'assurer des revenus aux destitués, pour ne pas les rendre économiquement dépendants des « organisations illégales ». Cf. Fribourg-en Brisgau, BA-MA, RW 36/224, p. 53.

82 BA-MA, RW 36/192, p. 73.

83 BA-MA, RW 36/224, p. 53.

84 Verordnungsblatt, $\mathrm{n}^{\circ} 34,8 / 3 / 1941$.

85 E. RASKIN, Gerard Romsée. Een ongewone man. Een ongewoon leven, Anvers, Hadewijch, 1995.

86 De benoemingen..., p. 13, dans AGR, Dossiers des magistrats, dossier n 2118 : Een groote tactische fout werd begaan met de verordening van 7 maart 1941 op de ouderdomsgrens niet uit te breiden tot de magistraten van den zetel, en ze zelfs niet volledig toe te passen voor wat de magistratuur van het parket betreft ».

87 BA-MA, RW 36/185, p. 52.

88 Tätigkeitsbericht des Chefs des Militärverwaltungsstabes, n 15, mars 1941 (BA-MA, RW 36/ 185, p. 76).

89 BA-MA, RW 36/382, p. 33.

$\underline{90}$ Ibidem, p. 39.

91 BA-MA, RW 36/400, p. 12.

$\underline{92}$ BA-MA, RW 36/382, p. 33.

93 BA-MA, RW 36/384, p. 46 et p. 76 et, surtout, Tätigkeitsbericht der Gruppe Justiz für den 
Monat März 1941 (BA-MA, RW 36/382, p. 26). Ce recours massif au règlement d'exception est justifié par l'absence d'une relève appropriée.

94 Chiffres à traiter avec précaution. Il ne s'agit là que d'une première estimation.

95 Cf. H. VAn Goethem, La convention de La Haye, la collaboration administrative en Belgique et la persécution des Juifs à Anvers, 1940-1942, dans Collaboration : Nazification ? Le cas du Luxembourg à la lumière des situations française, belge et néerlandaise, ?Actes du colloque international organisé par les Archives Nationales du Luxembourg et le CEGES?, Luxembourg, Archives Nationales, mai 2006, p. 142s.

$\underline{96}$ Ibid., p. 149-186.

97 Par exemple : E. Verhoeyen, La Belgique occupée, De l'an 40 à la Libération, Bruxelles, 1994, p. 85-88, et N. WOUTERS, De Führerstaat..., p. 156-160.

98 BA-MA, RW 36/401 et CEGESOMA, AA 1941/270.

99 Ces questions sont développées dans R. VAN DOORSLAER, De Belgische politie en magistratuur en het probleem van de ordehandhaving, dans België in de Tweede Wereldoorlog, éd. E. VERHOEYEN e. a., Kapellen, 1990, p. 100-123.

100 M. Bost et J. J. ZURNÉ, Excluding the wrongdoers : the use of judicial discipline as a purging tool of the Belgian magistracy after the two German occupations, dans M. BERGÈRE, J. CAMPION, E. Droit, D. Rigoll, M.-B. Vincent (dir.), Pour une Histoire connectée et transnationale des épurations en Europe au sortir de la Seconde Guerre mondiale, Bruxelles, Peter Lang, à paraître en 2018.

PDF généré automatiquement le 2020-07-07 08:35:10

Url de l'article : https://popups.uliege.be:443/1370-2262/index.php?id=397 